Bull. Mater. Sci., Vol. 16, No. 6, December 1993, pp. 685-692. (C) Printed in India.

\title{
Oxygen incorporation during in situ growth of YBCO films on both sides of substrates
}

\author{
S P PAI, R PINTO, P R APTE, C P D'SOUZA, A G CHOUREY \\ and D KUMAR* \\ Tata Institute of Fundamental Research, Homi Bhabha Road, Bombay 400005 , India \\ * Chemistry Department, Indian Institute of Technology, Powai, Bombay 400076, India \\ MS received 12 July 1993; revised 18 September 1993
}

\begin{abstract}
Oxygen out-diffusion during cooling and heating of in situ grown $\mathrm{YBa}_{2} \mathrm{Cu}_{3} \mathrm{O}_{7-\delta}$ (YBCO) films in low oxygen pressure used during growth by pulsed laser deposition was studied in the temperature range $700-450^{\circ} \mathrm{C}$ using in situ resistance measurements. Results indicate that irrespective of the number of cooling and heating cycles seen by the films, full oxygenation of the films can be realized by the final cooling from the growth temperature in 500 torr oxygen pressure. This result has been successfully used to sequentially grow high quality YBCO films on both sides of $\mathrm{LaAlO}_{3}$ substrates. These films have been used for the fabrication of $\mathrm{X}$-band microstrip resonators with superconducting ground plane.
\end{abstract}

Keywords. Pulsed laser deposition; in situ YBCO films; oxygen incorporation; microstrip resonators; YBCO ground plane.

\section{Introduction}

Oxygen out-diffusion and in-diffusion in $\mathrm{Y}_{1} \mathrm{Ba}_{2} \mathrm{Cu}_{3} \mathrm{O}_{7-\delta}$ (YBCO) compounds has been studied earlier at various temperatures (Tu et al 1988; Xie et al 1989). It is well known that the properties of $\mathrm{Y}_{1} \mathrm{Ba}_{2} \mathrm{Cu}_{3} \mathrm{O}_{7-\delta}$ compounds, especially the superconducting properties, depend critically upon the oxygen stoichiometry or the value of $\delta$ (Jorgensen et al 1987, 1990). The crystal is superconducting with orthorhombic structure with $\delta$ in the range $0.5>\delta>0$, and has a semiconducting tetragonal structure with $\delta$ in the range $1>\delta>0.5$. Thin films of YBCO, due to their large surface area compared to their thickness, are particularly sensitive to oxygen out-diffusion and in-diffusion (Kwok and Ying 1991; Yamamoto et al 1991). Hence, processes involving high temperature treatment greatly affect the oxygen stoichiometry of YBCO films.

One of the important processes which involves the heating of YBCO film is the in situ growth of a second YBCO film on the other side of a substrate which is desirable as a superconducting ground plane for microwave passive devices (Pinto et al 1993). In other words, realization of in situ YBCO film sequentially on both sides of a substrate involves heating of the film grown first. Two techniques have been reported in the literature for the growth of films on both sides of substrates. The first is the metal organic chemical vapour deposition (MOCVD) technique which, by its very nature, is advantageous for the realization of YBCO films on both sides of substrates (Takemoto et al 1991). However, the quality of the films realized with MOCVD is still not as good as the quality realizable by some physical vapour deposition techniques such as pulsed laser deposition (PLD) and off-axis magnetron sputtering. Furthermore, it is less convenient than the PLD technique. The second method for the in situ growth of YBCO film on both sides of substrates is as in 
Foltyn et al (1991). In this method, the PLD technique is used and the substrate is heated by radiation from a halogen lamp heating stage. The substrate does not come in contact with the heater face plate, and hence there is no contamination. However, one disadvantage is that since most of the single crystal substrates $\left(\mathrm{SrTiO}_{3}, \mathrm{MgO}\right.$, $\mathrm{LaAlO}_{3}$, etc.) used for the growth of YBCO films are near-transparent to radiation from halogen lamps, it is difficult, in the initial stages, to realize substrate temperature in the neighbourhood of $700^{\circ} \mathrm{C}$ required for YBCO film growth. Moreover, since the absorption of heat by the substrate changes substantially in the early stages as the film thickness increases, a closed loop feedback control is essential to control the substrate temperature.

Here we report a new technique for the in situ growth of YBCO films by PLD on both sides of $\langle 100\rangle \mathrm{LaAlO}_{3}$ substrates sequentially with a buffer substrate between the heater face plate and the $\mathrm{LaAlO}_{3}$ substrate. We have also studied oxygen out-diffusion from the films during thermal cycling between $700^{\circ} \mathrm{C}$ and $200^{\circ} \mathrm{C}$ using in situ resistance measurements. These results clearly indicate that irrespective of the number of heating and cooling cycles seen by the films, it is the final oxygenation in oxygen ambient that is important for realizing proper oxygen stoichiometry of the films. Hence, this behaviour of YBCO thin films is similar to that observed in bulk YBCO material.

\section{Experiment}

The in situ growth and in situ resistance measurements of YBCO films were carried out in a pulsed laser deposition system using $15 \mathrm{~mm}$ dia and $3 \mathrm{~mm}$ thick YBCO target. A $248 \mathrm{~nm} \mathrm{KrF}$ excimer laser with $10 \mathrm{~Hz}$ repetition rate and $3 \mathrm{~J} \mathrm{~cm}^{-2}$ energy density was used. The details of YBCO film growth by PLD are given elsewhere (Pinto et al 1992). The buffer substrate used in the experiments was $10 \mathrm{~mm} \times 10 \mathrm{~mm} \times$ $0.5 \mathrm{~mm} \mathrm{MgO}$ mounted on the heater face plate by high temperature silver paste. The device substrate, $10 \mathrm{~mm} \times 10 \mathrm{~mm} \times 0.5 \mathrm{~mm}\langle 100\rangle \mathrm{LaAlO}_{3}$, was mounted edge-toedge on the $\mathrm{MgO}$ buffer substrate using four stainless steel clips as shown in figure 1. The main purpose of the buffer substrate is to avoid contamination of the device substrate from the heater face plate. So the $\mathrm{MgO}$ substrate acts as clean buffer between the device substrate and the heater. Obviously, since YBCO films are to be grown on both sides of $\mathrm{LaAlO}_{3}$, silver paste could not be used to mount $\mathrm{LaAlO}_{3}$ on the MgO buffer.

One disadvantage of the $\mathrm{MgO}$ buffer is the increased temperature drop between the heater plate and $\mathrm{LaAlO}_{3}$ top surface. Temperature measurements carried out

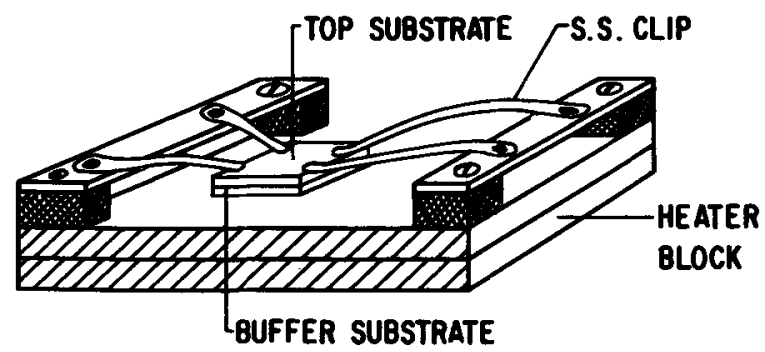

Figure 1. Schematic of substrate mounting with buffer $\mathrm{MgO}$ substrate. 
using thermocouples fixed with silver paste on heater face plate and $\mathrm{LaAlO}_{3}$ top surface have shown temperature differences of $120^{\circ} \mathrm{C}$ and $80^{\circ} \mathrm{C}$ between the substrate top surface and heater surface with and without $\mathrm{MgO}$ buffer, respectively. This magnitude of temperature difference was observed when the heater temperature was in the neighbourhood of $800^{\circ} \mathrm{C}$, and it is clear that the $\mathrm{MgO}$ buffer itself drops the temperature by $40^{\circ} \mathrm{C}$. This problem could, however, be solved by increasing the heater temperature appropriately to compensate for the drop due to $\mathrm{MgO}$ buffer. Although this means an additional strain on the heater, it is relatively minor compared to the advantage of the clean buffer in eliminating contamination of the substrate from the heater face plate.

In situ resistance measurements were carried out using Au wires fixed with silver paste on Ti-Pd-Au stripes deposited on the two ends of the $\mathrm{LaAlO}_{3}$ substrates. The substrates were mounted on the MgO buffer as before using electrically isolated stainless steel clips. The temperature was measured using a thermocouple fixed on the substrate using silver paste. A substrate temperature of $700^{\circ} \mathrm{C}$ and an $\mathrm{O}_{2}$ pressure of 0.2 torr were used during YBCO film growth. All the films studied had thicknesses in the range $2000-4000 \AA$.

\section{Results and discussion}

In situ resistance measurements during film growth as well as subsequent cooling or heating steps provide important information regarding the oxygen content of the film (Yamamoto et al 1991). This information is based on the correlation between the resistivity and the oxygen content of the $\mathrm{Y}_{1} \mathrm{Ba}_{2} \mathrm{Cu}_{3} \mathrm{O}_{7-\delta}$ film. This correlation has been studied earlier and most of the reported results show that the normal state resistivity is nearly proportional to $\delta /(1-\delta)$ (Cava et al 1987; Fiory et al 1987).

Figure 2 shows the resistance of in situ grown YBCO film during cooling and heating steps in two $\mathrm{O}_{2}$ pressures, 0.2 torr used during growth and 500 torr used during post-deposition oxygenation cooling. Figure $2 \mathrm{a}$ shows that the film resistance drops by more than an order of magnitude when it is cooled to $450^{\circ} \mathrm{C}$ in 500 torr $\mathrm{O}_{2}$. This is part of post-deposition oxygenation step. However, if the same film is reheated to $700^{\circ} \mathrm{C}$ in 0.2 torr oxygen used during growth, the film resistance rises almost to the original value in about $5 \mathrm{~min}$ required for the substrate to attain $700^{\circ} \mathrm{C}$. This obviously means that nearly all the additional oxygen incorporated in the filin during cooling has out-diffused during heating in 0.2 torr oxygen pressure. The subsequent step in figure $2 \mathrm{a}$ is the cooling of the film in 500 torr oxygen to $200^{\circ} \mathrm{C}$ which shows resistance drop by nearly two orders of magnitude indicating a transition to complete orthorhombicity. This was confirmed by X-ray diffraction (XRD) as described later. A further heating to $700^{\circ} \mathrm{C}$ in $0-2$ torr $\mathrm{O}_{2}$ almost restores the original growth resistance indicating out-diffusion during heating. However, a further cooling in 500 torr oxygen once again restores the orthorhombic structure in the film as seen by a sharp drop in resistance by nearly two orders of magnitude.

If the film is cooled to $450^{\circ} \mathrm{C}$ in 0.2 torr $\mathrm{O}_{2}$ instead of cooling it in 500 torr $\mathrm{O}_{2}$, there is no appreciable change in resistance as seen in figure $2 \mathrm{~b}$. However, the resistance increases slightly if the film is reheated to $700^{\circ} \mathrm{C}$ in 0.2 torr oxygen indicating a slight out-diffusion of oxygen. We believe that a slight $\mathrm{O}_{2}$ out-diffusion has also taken place during cooling to $450^{\circ} \mathrm{C}$ in 0.2 torr oxygen as the resistance has not changed from $700^{\circ} \mathrm{C}$ to $450^{\circ} \mathrm{C}$. It appears that the slight increase in resistance due to some oxygen 

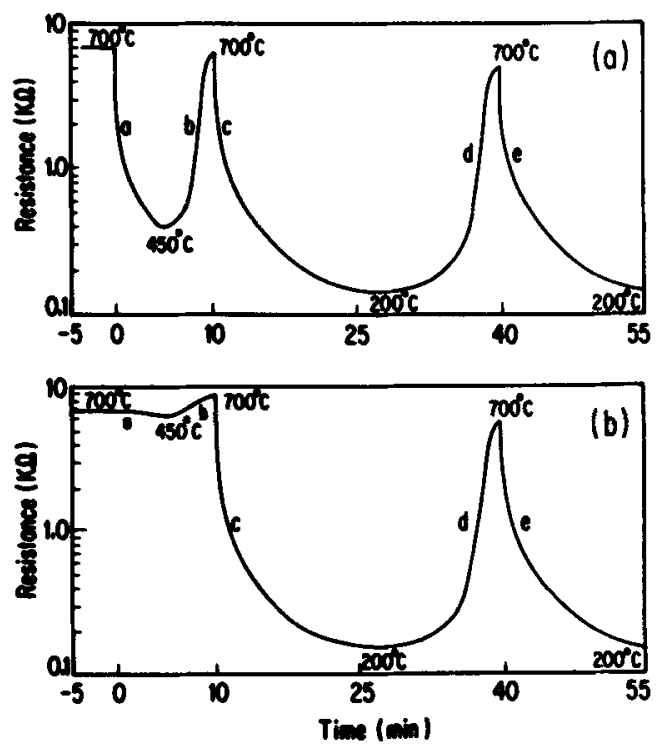

Figure 2. Variation of resistance with time measured on in situ grown YBCO films during (a) cooling in 500 torr oxygen (steps a, c and e) and heating in 0-2 torr oxygen (steps b and d), and (b) cooling in 0.2 tor oxygen (step a) and in 500 torr oxygen (steps $c$ and e) and heating in 0.2 torr oxygen (steps $b$ and d).

out-diffusion is compensated by a slight drop in resistance from $700^{\circ} \mathrm{C}$ to $450^{\circ} \mathrm{C}$. What is interesting, however, is the restoration of complete orthorhombicity in the film by cooling from $700^{\circ} \mathrm{C}$ to $200^{\circ} \mathrm{C}$ in 500 torr $\mathrm{O}_{2}$. Subsequent heating in 0.2 torr oxygen and cooling in 500 torr oxygen reproduce the nature of oxygen out-diffusion and in-duffusion, respectively, similar to that shown in figure $2 a$.

From the in situ resistance measurement experiments described above it is clear that irrespective of the number of cooling and heating steps and irrespective of the number of oxygen in-diffusion and out-diffusion cycles seen by the in situ grown YBCO film, the last oxygenation cooling step in 500 torr oxygen is essential for the realization of orthorhombicity and hence superconductivity in the film. The above result is extremely important for the in situ growth of YBCO films sequentially on both sides of substrates. Therefore, we have used this result for realizing high quality YBCO films on both sides of $\langle 100\rangle \mathrm{LaAlO}_{3}$ substrates by in situ growth sequentially. Hence, as mentioned earlier, the $\mathrm{MgO}$ buffer substrate was found to be essential in eliminating contamination on the $\mathrm{LaAlO}_{3}$ substrate from the heater plate. The YBCO film was first in situ grown on one side of the $\mathrm{LaAlO}_{3}$ substrate followed by the normal oxygenation step in 500 torr oxygen. The crystalline quality of the film is shown in figure $3 \mathrm{a}$ which shows the XRD spectrum of the film. As seen in the figure, the film is nearly epitaxial with a high degree of $c$-axis orientation and $c$-axis length of $11.71 \AA$. In order to grow YBCO film on other side, the $\mathrm{LaAlO}_{3}$ substrate was remounted on the buffer $\mathrm{MgO}$ edge-to-edge with the side having YBCO film in contact with the buffer $\mathrm{MgO}$. The growth chamber was then evacuated followed by heating of the substrate to $700^{\circ} \mathrm{C}$ in 0.2 torr oxygen. It may be noted here that a substrate top surface temperature of $700^{\circ} \mathrm{C}$ means a bottom temperature of $740^{\circ} \mathrm{C}$ which is the temperature seen by the film. Obviously, oxygen would out-diffuse from this film 


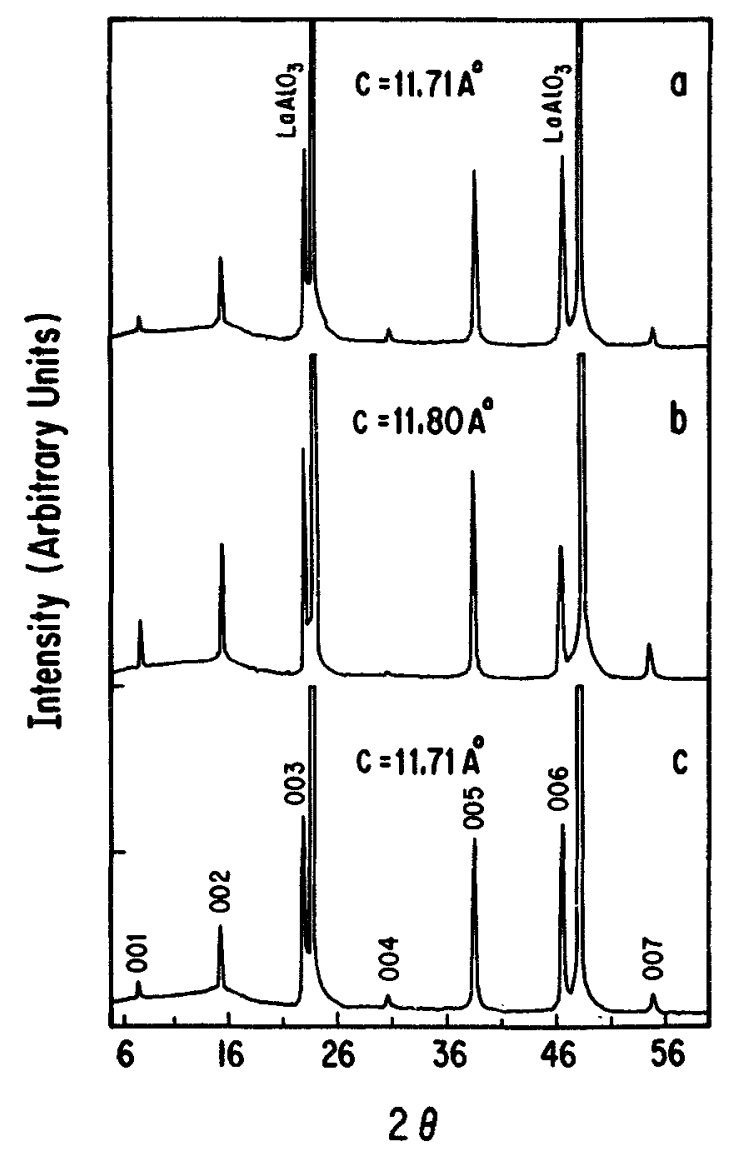

Figure 3. X-ray diffraction spectra of YBCO films at various stages: (a) in situ grown and cooled at 500 torr oxygen from $700^{\circ} \mathrm{C}$ to room temperature; (b) heated to $700^{\circ} \mathrm{C}$ in 0.2 torr oxygen and cooled subsequently in 500 torr nitrogen with 0.2 torr oxygen partial pressure; and (c) heated to $700^{\circ} \mathrm{C}$ in 0.2 torr oxygen and cooled in 500 torr oxygen.

during the heating stage as shown in figure 2 . In order to find the crystallinity of the film at this stage we cooled the film to room temperature in 500 torr nitrogen retaining 0.2 torr partial pressure of oxygen and obtained an XRD spectrum. Shown in figure $3 \mathrm{~b}$ is the XRD spectrum of this film. It is clear that the film is $c$-axis oriented with an expanded $c$-axis length of $11.80 \AA$ indicating an oxygen deficient orthorhombic structure. The substrate was then remounted on the buffer $\mathrm{MgO}$ with the side having YBCO film in contact with the buffer. Then the chamber was once again evacuated followed by heating to attain $700^{\circ} \mathrm{C}$ substrate temperature in 0.2 torr oxygen. The second film was then in situ grown as before followed by cooling in 500 torr oxygen for oxygenation of the films. The XRD spectrum of the first film was then obtained again. Shown in figure $3 c$ is the XRD spectrum of the film grown first which was reheated and finally cooled down in 500 torr oxygen. It is evident that this spectrum is nearly identical to that shown in figure $3 \mathrm{a}$ indicating that $c$-axis length of $11.71 \AA$ is restored.

Further characterization of the films grown sequentially on both sides of $\mathrm{LaAlO}_{3}$ was carried out by measuring transition temperature for zero resistance, $T_{c}$, and 


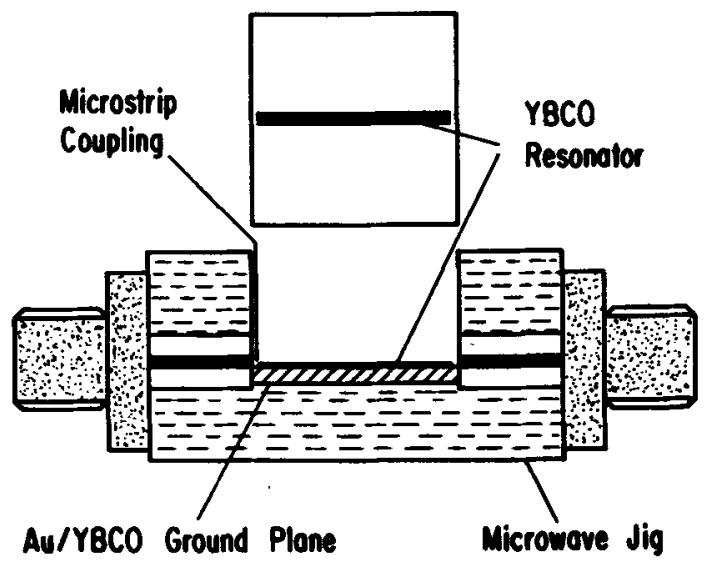

Figure 4. Schematic of X-band microstrip resonator with mounting jig.

critical current density, $J_{c}$ (zero magnetic field). It was found that the $T_{c}$ of the films was practically the same, $88-89 \mathrm{~K}$. $J_{c}$ was measured in many films with $\sim 2000 \AA$ thickness and patterned into microbridges. It was found that the films grown first showed $J_{c}$ in the range $1.5-2.2 \times 10^{6} \mathrm{~A} \mathrm{~cm}^{-2}$ at $77 \mathrm{~K}$. The films grown second, however, showed $J_{c}$ in a wider range, $1.5-3.5 \times 10^{6} \mathrm{~A} \mathrm{~cm}^{-2}$ at $77 \mathrm{~K}$. Nevertheless, this difference in $J_{c}$ is small and hence negligible. Hence, it is clear that the low frequency transport properties of films grown on both sides are excellent.

As mentioned earlier, the main purpose of growing films on both sides of $\mathrm{LaAlO}_{3}$ is to realize superconducting ground plane for microstrip resonators operating at microwave frequencies (Pinto et al 1993). Besides resonators, superconducting ground plane is also desirable for other microwave passive devices such as filters and delay lines (Zahopoulos et al 1991). We have carried out extensive work on the effect of ground plane on the measured $Q$-factor, $Q_{\text {meas }}$, of thin film YBCO microstrip resonators at $\mathrm{X}$-band frequencies. Shown in figure 4 is a schematic of an X-band microstrip resonator. It is a half-wavelength resonator with input/output couplings through capacitive gaps between the YBCO transmission line and the SMA connector pins. Both the device ports were loosely coupled such that $Q_{\text {meas }}$ is approximately equal to the unloaded $Q$-factor, $Q_{0}$, of the resonator (Lancaster et al 1992). The microwave measurements were carried out using HP8757C scalar network analyser and HP83620A synthesized sweeper with the associated reflectometry set-up. The low temperature measurements were carried out by mounting the resonator on the cold head of a closed cycle He cryocooler with a temperature range $10-300 \mathrm{~K}$. Further details of microwave measurement set-up are reported elsewhere (Pinto et al 1993).

It may be noted here that in the absence of the superconducting YBCO ground plane, the $50 \mu \mathrm{m}$ thick $\mathrm{Au}$ foil $(10 \mathrm{~mm} \times 10 \mathrm{~mm})$ placed between the $\mathrm{LaAlO}_{3}$ device substrate and the gold plated microwave jig (shown in figure 4) acts as an Au ground plane. The resonator $Q$-factors were measured by calculating the ratio $f / \Delta f$, where $f$ is the centre frequency and $\Delta f$ is $3 \mathrm{~dB}$ bandwidth of resonant curves.

Results have shown that the superconducting ground plane improves the measured $Q$-factor by about $30 \%$ at $77 \mathrm{~K}$. For example, the typical $Q$-factor obtained for thin film YBCO X-band microstrip resonator on $\mathrm{LaAlO}_{3}$ at $77 \mathrm{~K}$ is $\sim 2800$ without superconducting ground plane, i.e. with $\mathrm{Au}$ ground plane. With a second YBCO thin 


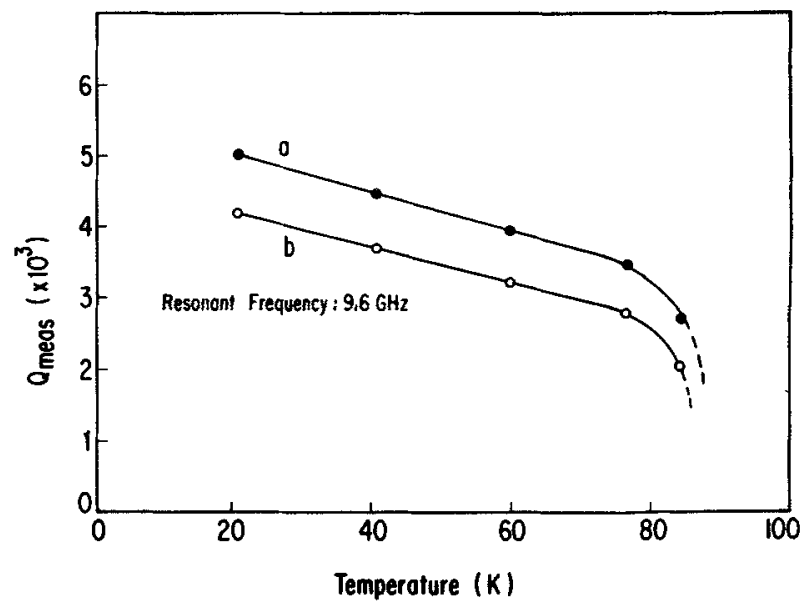

Figure 5. Variation of $Q_{\text {meas }}$ with temperature obtained for X-band YBCO microstrip resonators: a. with superconducting $\mathrm{YBCO}$ ground plane and $\mathrm{b}$. with Au ground plane.

film on the back of the $\mathrm{LaAlO}_{3}$ substrate acting as a superconducting ground plane the $Q$-factor was found to increase to $\sim 3600$ at $77 \mathrm{~K}$. The thickness of both the YBCO films in this case is $\sim 4000 \AA$. Shown in figure 5 are variations of $Q_{\text {meas }}$ (or $Q_{0}$ ) with temperature obtained for YBCO thin film microstrip resonators with superconducting YBCO and Au ground plane. It should be noted here that a further improvement in measured $Q$-factor is limited by the loss tangent, $\tan \delta$, of $\mathrm{LaAlO}_{3}$ substrate, which is $\sim 10^{-4}$ (Lancaster et al 1992). Nevertheless, an improvement in $Q$-factor from 2800 to 3600 at $77 \mathrm{~K}$ realized with the incorporation of a superconducting ground plane is significant.

\section{Summary}

Oxygen incorporation in the in situ grown YBCO films depends upon the oxygen pressure used during cooling. By in situ resistance measurements we have shown that there is substantial out-diffusion of oxygen from the YBCO films in situ grown by PLD if they are cooled or heated in the $700-450^{\circ} \mathrm{C}$ temperature range in low oxygen pressure of 0.2 torr used during growth. However, irrespective of the number of cooling and heating steps seen by the YBCO film in 0.2 torr oxygen, the final oxygenation step of cooling from the growth temperature in 500 torr oxygen has been found to be important in restoring orthorhombicity and hence superconductivity in the film. So, this property of YBCO thin films is similar to the behaviour of bulk YBCO material. This result has been used for the in situ growth of YBCO films sequentially on both sides of $\mathrm{LaAlO}_{3}$ substrates using a buffer $\mathrm{MgO}$ substrate to eliminate contamination on $\mathrm{LaAlO}_{3}$ from the heater plate. XRD and $J_{c}$ measurements have indicated that the film grown first has high epitaxial quality similar to that of the film grown second. Furthermore, the second YBCO film has been used as a superconducting ground plane for thin film YBCO microstrip resonators operating at X-band frequencies. Results have shown that the measured $Q$-factor of X-band microstrip resonators with $\mathrm{Au}$ ground plane improves from 2800 to 3600 at $77 \mathrm{~K}$ with the incorporation of superconducting YBCO ground plane on $\mathrm{LaAlO}_{3}$ substrates. 


\section{Acknowledgements}

The authors thank Professors R Vijayaraghavan, L C Gupta and M Sharon for useful discussions.

\section{References}

Cava R J, Batlogg B, Chen C H, Rietman E A, Zahurak S M and Werder D 1987 Phys. Rev. B36 5719 Fiory A T, Gurvitch M, Cava R J and Espinosa G P 1987 Phys. Rev. B36 7262

Foltyn S R, Muenchausen R E, Dye R C, Wu X D, Luo L, Cooke D W and Taber R C 1991 Appl. Phys. Lett. 591374

Jorgensen J D et al 1987 Phys. Rev. B36 3608

Jorgensen J D, Veal B W, Paulikas A P, Crabtree G W, Clauss H and Kwok W K 1990 Phys. Rev. B42 1863

Kwok H S and Ying Q Y 1991 Physica C177 122

Lancaster M J, Maclean T S M, Wu Z, Porch A, Woodall P and Alford N NcN 1992 IEE Proc. H139 149

Pinto R, Pai S P, D'Souza C P, Gupta L C, Vijayaraghavan R, Kumar D and Sharon M 1992 Physica C196 264

Pinto R, Apte P R, Goyal N, Gupta L C, Vijayaraghavan R, Easwar K and Sarkar B K 1993 Appl. Supercond. 11

Takemoto J H, Jackson C M, Manasevit H M, St John D C, Burch J F, Daly K P and Simon R W 1991 Appl. Phys. Lett. 581109

Tu K N, Tsuei C C, Park S I and Levi A 1988 Phys. Rev. B38 772

Xie X M, Chen T G and Wu Z L 1989 Phys. Rev. B40 4549

Yamamoto K, Lairson B M, Bravman J C and Geballe T H 1991 J. Appl. Phys. 697189

Zahopoulos C, Sridhar S, Bautista J J, Ortiz G and Lanagan M 1991 Appl. Phys. Lett. 58977 